\title{
Frecuencia relativa de carcinoma escamoso y adenocarcinoma esofágicos en una serie de biopsias endoscópicas realizadas en Rosario, Argentina
}

\author{
Ariel Enrique Naves, ${ }^{1}$ Florencia Lapalma ${ }^{2}$ y José Ignacio Rodríguez Ciancio ${ }^{2}$
}

Forma de citar

Naves AE, Lapalma F, Rodríguez Ciancio JI. Frecuencia relativa de carcinoma escamoso y adenocarcinoma esofágicos en una serie de biopsias endoscópicas realizadas en Rosario, Argentina. Rev Panam Salud Publica. 2007;22(6):383-8.

RESUMEN Objetivos. Determinar en una serie de biopsias endoscópicas esofágicas consecutivas registradas en un laboratorio de anatomía patológica de la ciudad de Rosario, Argentina, la frecuencia relativa de adenocarcinoma y carcinoma escamoso, comparando los períodos 1992-1999 y 2000-2006. Verificar si se observa un aumento en la frecuencia relativa de adenocarcinoma esofágico con respecto al carcinoma escamoso, similar al notificado en otros países occidentales.

Métodos. Se estudiaron las biopsias endoscópicas esofágicas con diagnóstico de adenocarcinoma y carcinoma escamoso infiltrantes, y de esófago de Barrett (EB) realizadas entre 1992 y 2006. Se compararon las frecuencias relativas de estos cánceres en los períodos 1992-1999 y 2000-2006 por la prueba de la Z y se analizó la distribución por edad y sexo mediante la prueba de la $\chi^{2}$, con un nivel de significación $(\alpha)$ de 0,05 .

Resultados. Se detectaron, en total, 125 carcinomas escamosos y adenocarcinomas infiltrantes. La frecuencia relativa adenocarcinoma/carcinoma escamoso fue 0,33/0,67 en toda la serie, 0,28/0,72 en el período 1992-1999 y 0,38/0,62 en el período 2000-2006. Las diferencias no fueron estadísticamente significativas.

Los hombres representaron $75,6 \%$ de los casos de adenocarcinoma y 57,1\% de los de carcinoma escamoso; esta diferencia resultó significativa $(\mathrm{P}<0,05)$. La edad media de los pacientes en el momento del diagnóstico era de 66,0 años en el caso del adenocarcinoma y 67,9 años en el del carcinoma escamoso. La frecuencia de carcinomas infiltrantes fue mayor entre los hombres menores de 65 años que entre las mujeres del mismo grupo etario $(\mathrm{P}<0,025)$. El EB se diagnosticó en una proporción de 6 a 1 con respecto al adenocarcinoma.

Conclusiones. El adenocarcinoma y el carcinoma escamoso predominaron en hombres, pero el adenocarcinoma en mayor proporción. La frecuencia relativa del adenocarcinoma aumentó en $10 \%$ en los últimos siete años de la serie con respecto a los primeros siete años. Esta diferencia no resultó significativa, pero puede representar una tendencia en nuestro medio similar a la descrita en otros países.

Palabras clave Esófago, biopsia, adenocarcinoma, carcinoma de células escamosas, Argentina.

1 Instituto de Histopatología, Rosario, Argentina. La correspondencia debe dirigirse a Ariel Enrique Naves, Instituto de Histopatología, Montevideo 1788, (2000) Rosario, Provincia de Santa Fe, Argentina; tel.: +54 (341) 421-0606, fax: +54 (341) 4402281, correo electrónico: arielenaves@yahoo.com.ar

2 Instituto Universitario Italiano de Rosario
En los últimos años se ha registrado un aumento en la incidencia del adenocarcinoma esofágico con respecto al carcinoma escamoso - en cifras absolutas y en frecuencia relativa- en va- rios países occidentales. En los Estados Unidos se ha llegado a considerar que se ha producido un aumento "epidémico" del número de casos de adenocarcinoma esofágico (1) —con incre- 
mentos anuales comprendidos entre el $4 \%$ y el $10 \%$ en los hombres durante el período 1976-1987-. En algunas series, la frecuencia del adenocarcinoma esofágico incluso superó a la del carcinoma escamoso esofágico $(2,3)$.

Durante el mismo período, la incidencia del carcinoma escamoso esofágico permaneció estable o incluso disminuyó en relación con las décadas anteriores (4).

En Canadá, se observó un aumento de la incidencia anual del adenocarcinoma de esófago distal entre 1964 y 2002, tanto en hombres (incremento anual medio del 9,5\%) como en mujeres (incremento anual medio del $4,3 \%$ ). La incidencia del carcinoma escamoso en el mismo período permaneció estable (5).

Como reflejo de estos cambios, la frecuencia relativa del carcinoma escamoso con respecto al adenocarcinoma fue decreciendo. Así, en una serie estadounidense, dicha frecuencia disminuyó de 2:1 a 1,2:1 entre 1988 y 1994 (6).

Estos adenocarcinomas emergentes tienen alta prevalencia en hombres, pero son relativamente raros en mujeres (7).

La explicación más probable del aumento de la incidencia del adenocarcinoma y la base fenotípica para su desarrollo es su relación con el esófago de Barrett (EB) como lesión precursora o asociada. Se ha afirmado que la prevalencia de este "carcinoma de Barrett" se ha incrementado rápidamente en los últimos años (8).

No se dispone de datos derivados de series amplias de la ciudad argentina de Rosario (Provincia de Santa Fe, Argentina) sobre las frecuencias relativas del carcinoma escamoso y el adenocarcinoma esofágico, ni sobre la distribución de los pacientes por sexo y edad en el momento del diagnóstico. Tampoco se ha determinado si en dicha ciudad se ha producido una variación en la frecuencia relativa de estos cánceres similar a la hallada en diversos países occidentales (9).

Los objetivos de este trabajo fueron: a) determinar, en una serie de biopsias endoscópicas esofágicas consecutivas registradas en un laboratorio de anatomía patológica de la ciudad de Rosa- rio, las frecuencias relativas de carcinoma escamoso y de adenocarcinoma de esófago, relacionando los datos con la edad y el sexo de los pacientes, b) investigar si existió una variación en la frecuencia relativa de ambos tipos de carcinoma entre los primeros y los últimos años de la serie, y c) cuantificar en la misma población los casos de EB con o sin displasia diagnosticados histopatológicamente.

\section{MATERIALES Y MÉTODOS}

Se revisaron los registros de archivo de todas las biopsias endoscópicas de esófago estudiadas en el Instituto de Histopatología de Rosario entre 1992 y 2006, y se consignaron los casos de adenocarcinoma esofágico, carcinoma escamoso esofágico, EB sin displasia y con displasia, lesión escamosa intraepitelial y otros tumores esofágicos. En cada caso, se registró el sexo y la edad del paciente. Los pacientes estudiados eran ambulatorios, estaban afiliados a organismos de medicina prepaga o a obras sociales, y pertenecían en su mayor parte a la clase media baja o la clase media típica (10). Se reevaluaron los diagnósticos dudosos o incompletos, consultando los protocolos completos originales o reexaminando los preparados histológicos archivados.

Se excluyeron los pacientes menores de 18 años, los registrados en el archivo con diagnósticos que no encuadraban en las categorías previamente enumeradas y aquellos con diagnóstico dudoso para los que no se contó con protocolos completos o material histológico para reevaluar.

Cuando para un mismo paciente se había registrado en el archivo más de un estudio biópsico, la clasificación para la evaluación final se basó en la lesión más avanzada presentada en el curso de la evolución.

Los diagnósticos de adenocarcinoma y carcinoma escamoso se consideraron como tales solamente en los casos de neoplasia infiltrante.

Los carcinomas infiltrantes poco diferenciados o indiferenciados, que no pudieron clasificarse claramente como adenocarcinoma o carcinoma esca- moso, se agruparon dentro de la categoría "otros tumores", como carcinomas indiferenciados.

Para el diagnóstico de EB se fijó como requisito el criterio de existencia de metaplasia intestinal del esófago documentada histológicamente (11), considerándola presente cuando se hallaron claramente células caliciformes, confirmadas por tinción con azul alciano a $\mathrm{pH}$ 2,5 en casos paucicelulares. El diagnóstico y la graduación de la displasia se realizaron según los criterios revisados por un consenso internacional (12).

Se describieron las frecuencias relativas de adenocarcinoma y carcinoma escamoso infiltrantes en toda la serie y en los períodos 1992-1996 y 20022006, y se realizó una comparación por medio de la prueba $Z$ y se analizó la distribución por edad y sexo mediante la prueba de la $\chi^{2}$, con un nivel de significación $(\alpha)$ de 0,05 .

En doce casos de adenocarcinoma, 25 casos de carcinoma escamoso y 54 casos de EB sin displasia faltaron datos sobre la edad del paciente. Los resultados relativos a la edad se obtuvieron tomando en cuenta solamente los casos con edad conocida. Las comparaciones se realizaron por medio de la prueba de la $\chi^{2}$.

Los pacientes con EB con o sin displasia sometidos a más de un estudio biópsico se incluyeron en la serie general con el diagnóstico más avanzado que presentaron en el curso de la evolución, pero se analizaron además por separado, para determinar el comportamiento de las lesiones durante el seguimiento.

Los análisis estadísticos se realizaron con el programa SPSS (Statistical Product and Service Solutions), versión 15.5.

\section{RESULTADOS}

En el período 1992-2006 se realizaron en el Instituto de Histopatología de la ciudad de Rosario 3655 biopsias endoscópicas esofágicas. A partir de estas biopsias, se diagnosticaron 41 adenocarcinomas infiltrantes y 84 carcinomas escamosos infiltrantes. Cuando se analizaron por separado 
CUADRO 1. Casos de adenocarcinoma y carcinoma escamoso infiltrantes de esófago registrados entre 1992 y 2006 en el Instituto de Histopatología, Rosario, Argentina

\begin{tabular}{lcccc}
\hline \multicolumn{1}{c}{ Período } & $\begin{array}{c}\text { Adenocarcinoma } \\
(\mathrm{A})\end{array}$ & $\begin{array}{c}\text { Carcinoma escamoso } \\
(\mathrm{CE})\end{array}$ & Total & $\begin{array}{c}\text { Frecuencia relativa } \\
(\mathrm{A} / \mathrm{CE})\end{array}$ \\
\hline $1992-1999$ & 18 & 46 & 64 & $0,28^{\mathrm{a}} / 0,72^{\mathrm{b}}$ \\
$2000-2006$ & 23 & 38 & 61 & $0,38^{\mathrm{a}} / 0,62^{\mathrm{b}}$ \\
Total: $1992-2006$ & 41 & 84 & 125 & $0,33 / 0,67$ \\
\hline
\end{tabular}

a, b $P=0,3422$ (prueba de la $Z$, diferencia no significativa).

CUADRO 2. Distribución por sexo de los casos de adenocarcinoma y carcinoma escamoso esofágicos infiltrantes registrados entre 1992 y 2006 en el Instituto de Histopatología, Rosario, Argentina

\begin{tabular}{lccccr}
\hline & \multicolumn{2}{c}{ Adenocarcinoma } & \multicolumn{2}{c}{ Carcinoma escamoso } & \\
\cline { 2 - 5 } & No. & $\%$ & No. & $\%$ & Total \\
\hline Hombres & 31 & $75,6^{\mathrm{a}}$ & 48 & $57,1^{\mathrm{a}}$ & 79 \\
Mujeres & 10 & $24,4^{\mathrm{b}}$ & 36 & $42,9^{\mathrm{b}}$ & 46 \\
Total & 41 & 100,0 & 84 & 100,0 & 125 \\
\hline
\end{tabular}

${ }_{\mathrm{a}, \mathrm{b}} \chi^{2}=4,03995175261132, P<0,05$ (diferencia estadísticamente significativa).

los datos relativos a los períodos 19921999 y 2000-2006, se encontraron los resultados presentados en el cuadro 1. Las diferencias entre las frecuencias relativas de adenocarcinoma y de carcinoma escamoso entre estos dos períodos no resultaron significativas (prueba de la Z).

Al analizar la distribución por sexo de los casos de adenocarcinoma y carcinoma escamoso infiltrantes se hallaron los resultados presentados en el cuadro 2.

La variable "edad" se analizó mediante la prueba de normalidad de Kolmogorov-Smirnov, en la que se ob- tuvo un valor $P$ de 0,001 (distribución normal). La edad media de los pacientes en el momento del diagnóstico fue de 66,0 años en el caso del adenocarcinoma (desviación estándar [DE]: 14,6 años, edad mínima: 42 años, edad máxima: 91 años) y de 67,9 años en el caso del carcinoma escamoso (DE: 12,6 años, edad mínima: 42 años, edad máxima: 91 años).

Al clasificar los casos de carcinoma infiltrante (adenocarcinoma o carcinoma escamoso) por sexo y grupo etario (menos de 65 años y 65 años o más), se obtuvieron los resultados presentados en el cuadro 3.
En el mismo período, se identificaron 244 pacientes con diagnóstico de EB (6,7\% de las biopsias): 231 sin displasia (119 hombres y 112 mujeres) y 13 con displasia (todos hombres). En todos los casos de EB con displasia se había registrado la edad del paciente. El EB se halló en una proporción de 6 a 1 con respecto a los adenocarcinomas registrados durante el mismo período.

En $47(18,6 \%)$ de los 231 casos de diagnóstico inicial de EB sin displasia, se había realizado por lo menos una biopsia de seguimiento; en uno de ellos, la lesión había desaparecido durante el seguimiento, en tres, había

CUADRO 3. Distribución por sexo y grupo etario de los casos de carcinoma esofágico infiltrante (adenocarcinoma y carcinoma escamoso) registrados entre 1992 y 2006 en el Instituto de Histopatología, Rosario, Argentina

\begin{tabular}{|c|c|c|c|c|c|}
\hline & \multicolumn{2}{|c|}{ Edad $<65$ años } & \multicolumn{2}{|c|}{ Edad $\geq 65$ años } & \multirow[b]{2}{*}{ Total } \\
\hline & No. & $\%$ & No. & $\%$ & \\
\hline Hombres & 32 & $74,4^{a}$ & 27 & $49,1^{\mathrm{a}}$ & $59^{c}$ \\
\hline Mujeres & 11 & $25,6^{b}$ & 28 & $50,9^{b}$ & $39^{c}$ \\
\hline Total & $43^{c}$ & 100,0 & $55^{c}$ & 100,0 & $98^{c}$ \\
\hline
\end{tabular}


progresado a displasia (a los 45, 32 y 9 meses de seguimiento, respectivamente) y en los restantes, había permanecido sin cambios.

En cuatro de los trece casos de EB con displasia, se había realizado por lo menos una biopsia de seguimiento; en uno de ellos, la lesión había progresado a adenocarcinoma a los siete meses de seguimiento, y en tres, el cuadro no había variado durante el seguimiento.

Se contabilizaron, además, ocho casos de lesión escamosa intraepitelial, tres de bajo grado y cinco de alto grado.

Por último, en la categoría "otros tumores", se clasificaron siete carcinomas infiltrantes que no pudieron identificarse con seguridad como adenocarcinoma o carcinoma escamoso (tres de ellos con características de carcinoma de células pequeñas), y se agruparon como "carcinomas indiferenciados", dos papilomas, dos melanomas, dos leiomiomas, dos linfomas y un hemangioma capilar.

\section{DISCUSIÓN}

La incidencia del adenocarcinoma de esófago se ha incrementado rápidamente en las últimas tres décadas. Así, entre los hombres estadounidenses blancos, se produjo un aumento del $350 \%$ entre mediados de la década del setenta y 1994. En el mismo período, la incidencia anual de carcinoma escamoso por 100000 habitantes descendió de 3,4 a 2,2 (13).

En un estudio del American College of Surgeons se halló que la incidencia relativa del carcinoma escamoso respecto del adenocarcinoma decreció de 2:1 a 1,2:1 entre 1988 y 1994 (6).

Es probable que en la actualidad, entre los nuevos casos de carcinoma esofágico diagnosticados en los Estados Unidos y en otros países se encuentren más casos de adenocarcinoma que de carcinoma escamoso (14).

La incidencia del adenocarcinoma de esófago también ha aumentado rápidamente en Europa occidental a partir de la década del setenta; en contraste, la incidencia del carcinoma escamoso de esófago ha permanecido relativamente constante o ha disminuido (15).
El significativo aumento de la incidencia del adenocarcinoma de esófago en los países de estilo de vida occidental se ha atribuido, al menos en parte, a un aumento del sobrepeso y la obesidad en las poblaciones (4-6). En Irlanda, la incidencia de este adenocarcinoma se incrementó en un $38 \%$ entre 1995 y 2004, lo cual se asoció con un aumento del $67 \%$ de la prevalencia de obesidad en el mismo período (16). En un estudio de casos y controles realizado en dicho país, el $82 \%$ de los pacientes con adenocarcinoma de esófago eran obesos o presentaban sobrepeso, mientras que en la población de referencia esta proporción era del 59\% $(P<0,001)(16)$.

Además de la obesidad, otros factores pueden explicar los cambios en la frecuencia relativa del adenocarcinoma esofágico respecto del carcinoma escamoso, como la disminución del hábito de fumar y algunos cambios en los patrones de nutrición y de uso de medicaciones (4).

En Argentina no hay datos sobre la frecuencia relativa de adenocarcinoma y carcinoma escamoso esofágicos. Según el Atlas de mortalidad por cáncer en la República Argentina 19972001 (9), del total de 144274 casos de cáncer en hombres, $6760(4,7 \%)$ correspondieron a cáncer de esófago (no se discrimina entre adenocarcinomas y carcinomas escamosos); mientras que del total de 119704 casos en mujeres, $2783(2,3 \%)$ correspondieron a cáncer de esófago. En la provincia de Santa $\mathrm{Fe}$, donde se halla la ciudad de Rosario -que concentra la mayor población urbana de la provincia-, este cáncer representó la sexta causa de mortalidad por cáncer en hombres, mientras que entre las mujeres no figuró entre las primeras diez causas.

En esta serie de biopsias endoscópicas esofágicas realizadas consecutivamente en un laboratorio de histopatología de Rosario, la frecuencia relativa de adenocarcinoma y carcinoma escamoso de esófago $(0,33 / 0,67)$ es similar a la observada en series de otros países (17). En una serie estadounidense de 1986 el adenocarcinoma representó el $34 \%$ de todos los cánceres esofágicos (18).
Al separar los casos más antiguos (1992-1999) de los más recientes (20002006), se observó una variación de la frecuencia relativa, que pasó de 0,28/ 0,72 a 0,38/0,62 (cuadro 1). Esta diferencia, aunque no resulta estadísticamente significativa, puede indicar una tendencia en nuestro medio similar, aunque menos acentuada, a la descrita en los Estados Unidos y en países de Europa occidental.

Si bien la frecuencia relativa en biopsias endoscópicas no es una medida directa de la incidencia ni de la prevalencia del adenocarcinoma y del carcinoma escamoso esofágico en la población general, estos datos permiten evaluar de manera indirecta el estado actual y la probable evolución de la frecuencia del adenocarcinoma esofágico entre personas de clase media baja y clase media típica de la ciudad de Rosario, teniendo en cuenta que derivan de estudios endoscópicos realizados en la misma población y por el mismo grupo de endoscopistas, y considerando que el diagnóstico inicial de adenocarcinoma y carcinoma escamoso esofágicos se realiza casi siempre por biopsia endoscópica. La similitud en el número total de carcinomas en los períodos 1992-1999 y 2000-2006 permite asimismo una comparación adecuada entre los grupos.

Algunos autores separan en sus series los casos de adenocarcinoma asociado a EB de los de adenocarcinoma cardial. En esta serie -que sólo incluye casos remitidos como biopsias esofágicas por endoscopistas - no realizamos esa diferencia, incluso si algunos de los casos podían considerarse como adenocarcinoma cardial, teniendo en cuenta que muchos autores atribuyen una patogenia similar a ambas lesiones y las consideran en conjunto (19).

En relación con el sexo, hallamos que en los hombres, el adenocarcinoma representa el 75,6\% de los casos y el carcinoma escamoso, el 57,1\%. La diferencia en la frecuencia de predominio masculino del adenocarcinoma respecto del carcinoma escamoso resultó estadísticamente significativa $(P<0,05)$. Esto es similar a lo descrito en series de Estados Unidos (7) e indica que aunque 
ambos tipos de carcinoma, de diferente etiopatogenia, son más frecuentes en el sexo masculino, la diferencia es mayor en el caso del adenocarcinoma.

En cuanto a la distribución por edad, encontramos que en las mujeres, la mayoría de los cánceres $(71,8 \%)$ se diagnosticaron a partir de los 65 años, mientras que en los hombres, algo más de la mitad de los diagnósticos $(54,2 \%)$ se realizaron antes de los 65 años $(P<$ $0,025)$ (cuadro 3). Estos resultados coinciden con la distribución de la mortalidad por cáncer de esófago según grupos etarios notificada en la Provincia de Santa Fe: el 57,7\% entre los varones mayores de 65 años y el 42,3\% entre los menores de 65 años, y el 83,3\% entre las mujeres mayores de 65 años y el 16,7\% entre las menores de 65 años (9).

El porcentaje de casos de EB diagnosticados en las biopsias endoscópicas esofágicas realizadas $(6,67 \%)$ es menor que el observado en pacientes asintomáticos de otras series (20). No obs- tante, cabe destacar que en esta serie se incluyeron numerosos casos en los cuales las biopsias se realizaron como parte de procedimientos de endoscopia digestiva alta por indicaciones ajenas a afección esofágica, sin estudio sistemático de la unión escamocolumnar.

El EB se diagnosticó en una proporción de 6 a 1 con respecto al adenocarcinoma. En la gran mayoría de los casos $(94,7 \%)$, no se observó displasia asociada en el momento del diagnóstico inicial. El número de casos de EB sin displasia fue similar en ambos sexos (119 hombres y 112 mujeres), lo que corresponde a una relación hombres:mujeres menor que la notificada, de 3:1 (21). En cambio, todos los casos de EB con displasia de esta serie se diagnosticaron en hombres. Ya se notificó anteriormente la alta predominancia masculina del EB, con mayor capacidad de progresión a displasia y cáncer (22).

Por último, sólo se realizaron biopsias de seguimiento en el 18,6\% de los

\section{REFERENCIAS}

1. Haggitt RC. Adenocarcinoma in Barrett's esophagus: a new epidemic? Human Pathol. 1992;23:475-6.

2. DeVault KR. Epidemiology and significance of Barrett's esophagus. Dig Dis. 2000-2001; 18:195-202.

3. Lambert R, Hainaut P. Esophageal cancer. World Gastroenterol News. 2006;11:37-9.

4. Enzinger PC, Mayer RJ. Esophageal cancer. N Engl J Med. 2003;349(23):2241-52.

5. Parfitt JR, Miladinovic Z, Driman DK. Increasing incidence of adenocarcinoma of the gastroesophageal junction and distal stomach in Canada-an epidemiological study from 19642002. Can J Gastroenterol.2006;20(4): 271-6.

6. Daly JM, Fry WA, Little AG, Winchester DP, McKee RF, Stewart AK, et al. Esophageal cancer: results of an American College of Surgeons patient care evaluation study. J Am Coll Surg. 2000;190:562-72.

7. Blot JW, Devesa SS, Kneller, RW, Fraumeni JF Jr. Rising incidence of adenocarcinoma of the esophagus and gastric cardia. JAMA. 1991; 265(10):1287-9.

8. Faller G, Stolte M. Barrett's oesophagus: time for consensus. Virchows Arch. 2003;443(5): 595-6.

9. Matos EL, Loria DI, Zengarini N, Fernández MM, Guevel CG, Marconi E, et al. Atlas de mortalidad por cáncer en Argentina, 19972001. Buenos Aires: Ministerio de Salud, Instituto de Oncología Ángel Roffo, Fundación Bunge y Born, y CPO-Piemonti; 2003.

10. Salvia A, Tami F (coordinadores). Barómetro de la deuda social argentina. N. ${ }^{\circ} 1$ : Las gran- des desigualdades. Buenos Aires: Universidad Católica Argentina; 2005.

11. Sharma P, McQuaid K, Dent J, Fennerty MB Sampliner R, Spechler S, et al. A critical review of the diagnosis and management of Barrett's Esophagus: The AGA Chicago Workshop. Gastroenterology. 2004;127:310-30.

12. Montogomery E, Bronner MP, Goldblum JR, Greenson JK, Haber MM, Hart J, et al. Reproducibility of the diagnosis of dysplasia in Barrett esophagus: a reaffirmation. Hum Pathol. 2001;32:368-78.

13. Devesa SS, Blot WJ, Fraumeni JF Jr. Changing patterns in the incidence of esophageal and gastric carcinoma in the United States. Cancer. 1998;83:2049-53. cer Working Group. Surgical resection with or without preoperative chemotherapy in oesophageal cancer: a randomised controlled trial. Lancet. 2002;359:1727-33.

15. Vaughan TL, Davis S, Kristal A, Thomas DB. Obesity, alcohol, and tobacco as risk factors for cancers of the esophagus and gastric cardia: adenocarcinoma versus squamous cell carcinoma. Cancer Epidemiol Biomarkers Prev. 1995;4(2):85-92.

16. Ryan AM, Rowley SP, Fitzgerald AP, Ravi N, Reynolds JV. Adenocarcinoma of the oesophagus and gastric cardia: male preponderance in association with obesity. Eur J Cancer. 2006; 42(8):1151-8.

17. Botterweck AA, Schouten LJ, Volovics A, Dorant E, van Den Brandt PA. Trends in the incidence of adenocarcinoma of the esophagus
14. Medical Research Council Oesophageal Can- casos de EB sin displasia, lo que sugiere que la proporción de casos que se controlan luego del diagnóstico de manera sistemática en nuestro medio es baja.

En resumen, en esta serie se registró un aumento del $10 \%$ en la frecuencia relativa del adenocarcinoma esofágico respecto del número total de casos de carcinoma escamoso y adenocarcinoma esofágicos en los últimos siete años, en comparación con los primeros siete años de la serie. Este aumento no resultó significativo, pero puede indicar una tendencia similar en nuestro medio a la descrita en otros países. $\mathrm{Si}$ bien tanto el adenocarcinoma como el carcinoma escamoso predominaron en hombres, el predominio masculino del adenocarcinoma fue mayor. En las mujeres, la mayoría de los cánceres se diagnosticaron después de los 65 años, mientras que en los hombres, más de la mitad de los diagnósticos se realizaron antes de los 65 años.

and gastric cardia in ten European countries. Int J Epidemiol. 2000;29:645-54.

18. Wang HH, Antonioli, DA, Goldman, H. Comparative features of esophageal and gastric adenocarcinomas: recent changes in type and frequency. Hum Pathol. 1986;17(5):482-7.

19. Chandrasoma $P$, Makarewicz K, Wickramasinghe $\mathrm{K}$, Demeester T. A proposal for a new validated histological definition of the gastroesophageal junction. Hum Pathol. 2006;37: 40-7.

20. Ward EM, Wolfsen HC, Achem SR, Loeb DS, Krishna M, Hemminger LL, et al. Barrett's esophagus is common in older men and women undergoing screening colonoscopy regardless of reflux symptoms. Am J Gastroenterol. 2006;101(1):12-7.

21. Falk GW, Thota PN, Richter JE, Connor JT, Wachserberger DM. Barrett's esophagus in women: demographic features and progression to high-grade dysplasia and cancer. Clin Gastroenterol Hepatol. 2005;3:1089-94.

22. Bani-Hani KE, Bani-Hani BK, Martin IG Characteristics of patients with columnarlined Barrett's esophagus and risk factors for progression to esophageal adenocarcinoma. World J Gastroenterol. 2005;11(43):6807-14.

Manuscrito recibido el 14 de diciembre de 2006. Aceptado para publicación, tras revisión, el 12 de septiembre de 2007. 
ABSTRACT Objectives. To determine the relative frequency of adenocarcinoma and squamous carcinoma in a series of endoscopic biopsies of the esophagus registered in consecutive order in a pathology laboratory in the city of Rosario, Argentina, during two time

Relative frequency of esophageal squamous carcinoma and adenocarcinoma in a series of endoscopic biopsies performed in Rosario, Argentina

Key words periods: 1992-1999 and 2000-2006. To determine if the relative frequency of esophageal adenocarcinoma has increased over that of squamous carcinoma, in keeping with the trends noted in other Western countries.

Methods. We studied the endoscopic esophageal biopsies diagnosed with infiltrating adenocarcinoma and squamous carcinoma and Barrett's esophagus (BE) between 1992 and 2006. The relative frequency of these cancers were compared for the periods 1992-1999 and 2000-2006 using the z-test. A distribution analysis for age and sex was performed by the chi-square test using a confidence level $(\alpha)$ of 0.05 .

Results. In all, 125 infiltrating squamous carcinomas and adenocarcinomas were found. The relative frequency of adenocarcinoma/squamous carcinoma for the entire series was 0.33/0.67; for the period from 1992-1990, 0.28/0.72; and from 2000-2006, $0.38 / 0.62$. The differences were not statistically significant. Males constituted $75.6 \%$ of the cases of adenocarcinoma and $57.1 \%$ of the cases of squamous carcinomas; this difference was statistically significant $(P<0.05)$. The mean age of the patients at the time of diagnosis was 66.0 years of age for adenocarcinoma and 67.9 years for squamous carcinoma. The frequency of infiltrating carcinoma was greater among the men than among the women of the age group under 65 years $(P<0.025)$. BE was diagnosed at a rate of $6: 1$ relative to adenocarcinoma.

Conclusions. Adenocarcinoma and squamous carcinoma were predominant among men, though adenocarcinoma was the major part of these. The relative frequency of adenocarcinoma rose $10 \%$ in the last 7 years of the series compared with the first 7 years. This difference was not significant, but it may signal a general trend similar to that described in other countries.

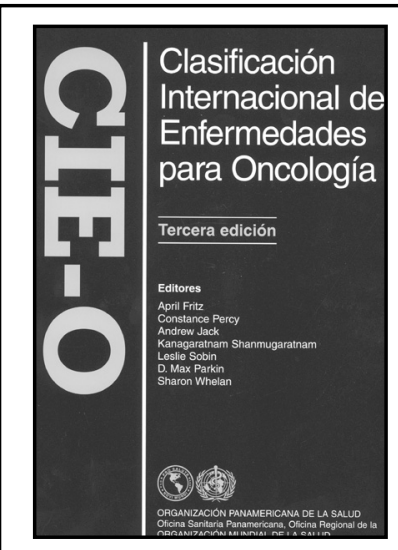

$2002 \cdot 320 \mathrm{pp}$.

ISBN 9275315868

Código: PC 586

Precio: US\$ 25.00 en América

Latina y el Carib / US\$ 34.00 en

el resto del mundo

\section{Clasificación Internacional de Enfermedades para Oncología (CIE-O) 3. a ed.}

La Clasificación Internacional de Enfermedades para Oncología (CIE-0) se ha usado durante casi 25 años como un instrumento estándar para codificar los diagnósticos de las neoplasias en los registros de tumores y de cáncer y en los laboratorios de anatomía patológica.

La CIE-0 es una clasificación dual con sistemas de codificación tanto para la topografía como para la morfología. El código topográfico describe el sitio de origen de la neoplasia y usa las mismas categorías de tres y cuatro caracteres que la sección de neoplasias del capítulo II de la CIE-10. El código morfológico describe las características del tumor mismo, incluidos su tipo de células y su actividad biológica. El índice alfabético presenta los códigos para la topografía y la morfología, e incluye aquellas lesiones y trastornos que se asemejan a los tumores.

En la última sección se presenta una guía para reconocer las diferencias de la codificación morfológica entre la segunda y la tercera edición. La guía comprende las listas de todos los códigos nuevos, los términos incorporados y los sinónimos agregados a las definiciones de los códigos existentes, la definición de los términos nuevos en los códigos de morfología, los términos que designan los trastornos que actualmente se consideran malignos, los términos eliminados y los términos que cambiaron en los códigos de comportamiento.

Usted también puede enviar su orden y pago en dólares estadounidenses a: http://publications.paho.org Fax: (301) 206-9789・E-mail: paho@pmds.com•Oficina de la OPS/OMS en su país 